Zbigniew Waleszczuk

Catholic University of Eichstaett-Ingolstadt, Germany

\title{
Die Familie - Relikt der Vergangenheit oder Fundament der Zukunft?
}

\section{The Family - a Relic of the Past or the Foundation for the Future?}

\begin{abstract}
This article is a description of the current situation of the family from a Christian perspective and is divided into three chapters according to the method of the catholic social teaching: see judge, act. In the first part, a analysis of reality is presented, that is a sociological view of the situation of the family in the European countries. The outline of the situation of the family is shown in three dimensions (socio-cultural, political and economic). The second part briefly treats the Catholic teaching on the family, especially their social significance according to John Paul II. The third part formulates practical conclusions to the question of what we can and should do to promote the family. It is the aim of Christian social ethics - as an instrument of evangelization to draw adequate conclusions resulting in effective actions of Christians. It is about building bridges between theology and the challenges of the present. Without a doubt, the family is not just a relic of the past, as it was provocatively formulated in the title, but it is, and will continue to be, the foundation of the future.
\end{abstract}

\section{Keywords}

Family; support; crisis; solidarity; future; John Paul II.

Die Frage des Themas scheint eine gewisse Provokation zu beinhalten, die uns einladen soll über die Bedeutung und Zukunft der Familie nachzudenken¹.

1 Dass die Christliche Gesellschaftslehre sich mit dem Wandel von Ehe und Familie auseinandersetzt, wird niemanden überraschen, der die kirchliche Sozialverkündigung kennt. Schon Ketteler war in den 1840er-Jahren empört über den Manchester-Kapitalismus, weil er die Familie in den Ruin treibe. Leo XIII. hielt 1891 in Rerum Novarum aus dem gleichen Grund Lohndumping für eine „Sünde, die zum Himmel schreit“". 
Man kann von dem „französischen“ Modell sprechen, das liberal und relativistisch geprägt ist, oder von dem ,irischen“, auf christlichen Wurzeln basierenden Modell, bei dem es selbstverständlich ist, dass die Ehe durch Mann und Frau geschlossen wird. Die gegenwärtigen Versuche, die Ehe als Partnerschaft gleichgeschlechtlicher Beziehungen mit allen Rechten und Pflichten der traditionellen Familie gleichzusetzen, bedeuten aus der Sicht der christlichen Sozialethik die Gefahr, die „normale“ Familie nur als eine Option unter vielen möglichen anzusehen und sie eher als Relikt der Vergangenheit zu betrachten. Gehört die Familie der Vergangenheit an? Wir können bemerken, dass die soziologischen Anzeichen, wie die Zahlen der Eheschließungen, Scheidungen usw., diese Tendenz zu bestätigen scheinen. Gleichzeitig ist sie immer noch der fundamentale Faktor des Glücks- und Geborgenheitsempfindens und steht immer noch ganz oben auf der Liste der erstrebenswerten Ziele bei jungen Menschen. Der Artikel beabsichtigt gemäß der Methode der Soziallehre (sehen, urteilen, handeln), das Thema in drei Schritten zu behandeln. Im ersten Teil versuchen wir eine Diagnose der Wirklichkeit zu formulieren, die Lage der Familie im heutigen Kontext darzustellen. Über die Situation der Familie gibt es derzeit zahlreiche Untersuchungen, wir begrenzen uns daher lediglich auf eine kurze Diagnose der Situation. In drei Perspektiven: soziokulturell, politisch und ökonomisch wollen wir die Lage der Familie besprechen. Im ersten Teil zeigen wir, ebenfalls skizzenhaft, die Soziallehre der Kirche in Bezug auf die Familie. Aufgrund der Tatsache, dass die Konferenz $z^{2}$ in Krakau stattgefunden hat, wenden wir unsere besondere Aufmerksamkeit der Lehre Johannes Pauls II. zu. Im dritten Teil versuchen wir praktische Handlungsvorschläge zu formulieren. Es geht um adäquate und effektive Antworten auf die gegenwärtigen Angriffe gegen Ehe und Familie. Damit unsere Handlungen eine wirksame Unterstützung der Familie sein können, geht es um die Suche nach konkreten Maßnahmen in allen oben genannten Bereichen, die der Familie wirkliche Räume für ihre Existenz und Entwicklung verschaffen, was letztendlich mit der Zukunft des Menschen zusammenhängt.

2 Der Vortrag über das Thema: Die Familie - Relikt der Vergangenheit oder Fundament der Zukunft?, wurde im Rahmen einer internationalen Konferenz an der Universität Johannes Paul II. in Krakau am 30.10.13 gehalten. 


\section{Zur Lage der Familie}

\section{Soziale Veränderungen und kulturelle Angriffe auf die Familie}

Die zentrale Kategorie der neuzeitlichen Ära ist die autonome Entfaltung der menschlichen Freiheit mit Hilfe der Philosophie, der Natur- und Sozialwissenschaften, sowie deren Anwendung in Technik, Ökonomie und Politik $^{3}$. „Die Menschheit erkennt sich - und das markiert geistesgeschichtlich den Beginn der Neuzeit - nicht mehr in einer vorgegebenen Ordnung, sondern in einer ihr aufgegebenen Dynamik. Alles erscheint möglich. Es entsteht im Vergleich zum Mittelalter eine neue Gesellschaft, deren wissenschaftlichtechnische, wirtschaftliche und politische Errungenschaften allmählich zur Entstehung eines allgemeinen Fortschrittsglaubens führten ${ }^{\text {“4 }}$. Durch die sprunghafte Entwicklung der Humanwissenschaften verbreitete sich das Gefühl, man könne nun auch das schwächste Glied der technologischen Fortschrittskette, nämlich den „unzuverlässigen Faktor Mensch“, wissenschaftlich im Sinne eines „Human Engineering“ endlich und endgültig in den Griff bekommen Wenige Jahre nach dem Ende des Zweiten Vatikanischen Konzils zeichnete sich allmählich eine Wende in der Betrachtung der Zukunftsperspektiven ab, die vom sehr radikalen Fortschritts-Optimismus abwich zu mehr Nüchternheit und sogar Nihilismus. Die vom Club of Rome 1972 formulierte These von den „Grenzen des Wachstums" ${ }^{\text {“6 }}$, die man als Krise des Fortschrittsglaubens bezeichnen kann, stellt die Frage: Wird es möglich sein, die zivilisatorische Entwicklung nach dem bisherigen Muster weiterzutreiben? Oder führt dies dazu, dass die Menschheit möglicherweise in eine Katastrophe treibt? ${ }^{7}$. Als Antwort auf die neuen Gefahren und Ängste der Zukunftsperspektiven entstand das Postulat der Reduzierung der menschlichen Population. Eine der Thesen, die als Angriff auf das bisherige Familienverständnis bezeichnet werden kann,

3 Siehe mehr: F.-X. Kaufmann, Die Krise der Kirche, Herder 2011.

4 L. Roos, Zwischen Hoffnung und Angst, „Die Neue Ordnung“ (2005) Nr. 6, S. 413.

5 A. Toffler, Der Zukunftsschock (Original: Future Shock, New York), Bern u. a. 1970.

6 Dennis Meadows u. a., Die Grenzen des Wachstums. Bericht des Club of Rome zur Lage der Menschheit, Stuttgart 1973.

7 Vgl. Dennis L. Meadows, Wachstum bis zur Katastrophe? Pro und Contra zum Weltmodell, Horst E. Richter (Hg.), Stuttgart 1974. 
ist die Verbreitung der Überbevölkerungstheorie ${ }^{8}$ und der Genderideologie ${ }^{9}$ mit ihren weitreichenden Folgen für die ganze Menschheitsfamilie. Diese Ideologien, die sich als Wissenschaft ${ }^{10}$ darstellen, werden heute in der globalisierten ${ }^{11}$ Welt schnell mit Hilfe der neuen Technologien, mit Politikund Wirtschaftsinstrumenten, oft gegen den Willen der lokalen Bevölkerung, nicht ohne Erfolg ${ }^{12}$, durchgesetzt.

Führt die gegenwärtige Entwicklung, die wir nur skizzenhaft darstellen können, zur Desorganisation, zur Auflösung der Institution Familie? Der neue Entwicklungsschub unserer Zeit stellt neue Fragen. Soziologisch können wir einige wichtige Veränderungen der Gegenwart folgendermaßen beschreiben:

- Mit zunehmender Erwerbstätigkeit der Frauen tritt die strukturelle Benachteiligung der Familie in die Wirtschafts- und Sozialordnung ein;

- Ehe und Elternschaft werden zunehmend nicht mehr als „natürliche“ Komponenten, als selbstverständlicher Regelfall für den Lebensweg des Menschen empfunden, sondern als eine unter mehreren „Optionen“, die in der individualisierten Gesellschaft als wählbar gegeben sind;

- Die mit der Gleichberechtigung der Frau einhergehende erhebliche Erweiterung ihrer Entfaltungsmöglichkeiten durch Ausbildung und Beruf;

- Die Entkoppelung von Sexualität und Fortpflanzung und die neuen Möglichkeiten der Familienplanung;

8 „1804 überschritt die Weltbevölkerung eine Milliarde Menschen und ein rapides Bevölkerungswachstum setzte ein. Innerhalb des 20. Jahrhunderts hat sich die Weltbevölkerung fast vervierfacht. 1927: 2 Milliarden, 1960: 3 Milliarden, 1974: 4 Milliarden, 1987: 5 Milliarden, 1999: 6 Milliarden und 2011: 7 Milliarden Menschen. Bei einem Bevölkerungswachstum von jährlich rund 79 Millionen Menschen steigt die Zahl der Erdenbürger in jeder Minute um 150 und jeden Tag um über 216.000. Angenommen, die Kinderzahl pro Frau bliebe bis 2100 konstant auf dem heutigen Niveau, würde die Weltbevölkerung zur Jahrhundertwende auf fast 27 Milliarden Menschen anwachsen“" UNESCO-Angaben, zitiert nach: Werner Mohrig: Wieviel Menschen trägt die Erde? Urania-Verlag, Leipzig/Jena/Berlin 1976, S. 10; Population Division of the Department of Economic and Social Affairs of the United Nations Secretariat (Hrsg.): World Population Prospects. The 2010 Revision. World Population change per year (thousands) Medium variant 1950-2050.

9 M. A. Peeters, Polityka globalistow przeciwko rodzinie, Warszawa 2013.

10 „Sie hat kaum etwas mit Wissenschaft zu tun, sondern ist eine Mischung aus diffusen Ängsten, Panikmache und rassistischen Grundelementen. Schon das Wort Überbevölkerung ist ja ein Unding. Das beinhaltet doch die Aussage, dass es irgendwo auf der Erde zu viele Menschen gibt und dass die weg müssen. Ich finde, Überbevölkerung ist ein emotionalisierender Begriff. [...]”. http://www. ener-gie.de/?p=902 (13.09.2013).

${ }^{11}$ Mehr über die Problematik und Dimensionen der Globalisierung, Siehe: Z. Waleszczuk, Globalisierung als neue Sozialfrage, Bayreuth 2004.

${ }^{12}$ Vgl. G. Kuby, Die globale sexuelle Revolution, Regensburg 2012. 
- Die Pluralisierung der Lebensformen und Liberalisierung des Verhaltens in Ehe und Familie;

- Der Rückgang der Heirats- und Geburtenquote (welcher die Frage nach der Zukunft des Sozial-Systems der Alterssicherung aufwirft);

- Die Zunahme der Ehescheidungen vermehrt die sozialerzieherischen Probleme („Scheidungswaisen“, Alleinerziehende) und stellt die Frage nach einem angemessenen Scheidungsrecht;

- Die Migration der jüngeren, gut ausgebildeten Menschen (z.B. aus Polen sind in den letzten Jahren 2 Millionen Menschen ins Ausland ausgewandert);

- Die zunehmende Infragestellung institutioneller Vorgaben der Gesellschaft durch nichteheliche Lebensgemeinschaften;

- ehelose Elternschaft;

- Demografische Entwicklung - Überalterung und Aussterben der Menschen in Europa;

- mediale Manipulationen;

- „Bildung eines neuen Menschen“ (in Polen liest z.B. 60\% der Gesellschaft keine Bücher mehr) ${ }^{13}$.

„Die Familie verliert für viele Menschen ihre zentrale Stellung; sie rückt an den Rand des gesellschaftlichen Lebens, weil sie die ökonomischen und sozialen Lebensvollzüge nicht mehr in sich integriert. Rechtlich beruht sie auf dem privaten Vertrag der Eheleute, ökonomisch treten Arbeitsort und privater Haushalt auseinander; die Familie ist nicht mehr wirtschaftliche Produktionseinheit“14. Die Familiensoziologie bezeichnet diesen Prozess, in

${ }^{13}$ Der neueste offizielle Rapport und die, durch die nationale Bibliothek durchgeführte Untersuchung zeigen eine erschreckende Tatsache. Der Bericht nennt sich „,soziale Reichweite des Buches in Polen im Jahr 2012”. http://bit.ly/19Ryz21 (11.10.2013). Hier einige Fakten aus diesem Bericht: nur 39\% der Polen haben überhaupt ein Buch in diesem Jahr gelesen, das bedeutet, dass $61 \%$ der Polen gar kein Buch gelesen haben. 34\% der Polen mit Hochschulausbildung haben im Jahre 2012 überhaupt kein Buch gelesen! 17\% dieser hochgebildeten Kreise lesen während eines Monats überhaupt keine Presse, 20\% der sogenannten polnischen Intelligenz sind außer Stande einen mehr als 3-seitigen Artikel im Monat zu lesen. Nur 11\% der Polen haben mindestens 7 Bücher im Jahr 2012 gelesen. Diese schockierenden Daten zeigen den sekundären Analphabetismus, die fortschreitende Verdummung und eine galoppierende Ignoranz. Und wenn jemand denkt, dass dies aufgrund der elektronischen Übertragung der Literatur geschieht, so liegt er falsch, denn der neueste Bericht der Nationalbibliothek stellt fest: ,, nur $2 \%$ der nicht Lesenden haben im Jahr 2012 überhaupt ein e-book gelesen". Vgl. R. Legutko, Triumf człowieka pospolitego, Warszawa 2012.

${ }^{14}$ B. Sutor, Politische Ethik, Paderborn 1991, S. 241. 
dem die Familie als privater Bereich der Gesellschaft an den Rand tritt, als ihre Desintegration ${ }^{15}$.

\section{2. Ökonomische Angriffe auf die Familie}

Die ökonomische Benachteiligung liegt zunächst darin, dass der Markt gegenüber der Familie blind ist, und zwar der Arbeits- wie auch der Gütermarkt. Wir können folgende Merkmale der gegenwärtigen liberal-individualistischen Ökonomie feststellen, die eine fehlende Sensibilität gegenüber der Bedeutung des Gemeinwohls und der Familie ${ }^{16}$ aufweisen:

- Der individuell gezahlte Leistungslohn nimmt keine Rücksicht darauf, ob der Empfänger nur für sich oder für eine Familie zu sorgen hat.

- Der Marktpreis der Güter ist der gleiche für alle Nachfrager, ob sie für eine oder für fünf Personen einkaufen.

- Verzichtet ein Ehepartner um der Kinder willen auf Erwerbstätigkeit, so verzichtet er nicht nur auf ein zusätzliches Einkommen, sondern zugleich auf die Steigerung seiner Ansprüche an die Rentenversicherung.

- Der Zeitaufwand der Eltern für Kinder und die Unterbrechung beruflicher Tätigkeit mindern ihre Wettbewerbs- und Karrierechancen im Beruf.

- Eine Familie mit zwei Kindern, in der ein Elternteil auf Erwerbstätigkeit verzichtet $^{17}$, fällt heute im Durchschnitt auf die Hälfte des im Vergleich allen Familien zur Verfügung stehenden Einkommens zurück. Kinder zu haben und zu erziehen wird also ökonomisch nicht honoriert, sondern ,bestraft ${ }^{\text {‘18 }}$.

${ }^{15}$ R. König, Die Rolle der Familie in der Soziologie, http://www.systemagazin.de/bibliothek/ texte/koenig_familiensoziologie.pdf (13.09.2013).

${ }^{16}$ B. Sutor, Politische Ethik, Paderborn 1991, S. 243.

17 „Wenn die Mutter ihren Beruf nicht aufgibt, was sie immerhin in der Hälfte aller Familien nicht tut, werden hohe Kosten für Kita oder Kinderbetreuung fällig. Und die schlagen umso heftiger zu Buche, wenn auch die anderen Kinder noch betreut werden müssen“. „So entgeht der Familie viel Einkommen, was die arbeitenden Väter nicht immer ausgleichen können. Auch diese entgangenen Einnahmen, die Opportunitätskosten, müssen Familien mitzählen, wenn sie die Rechnung für oder gegen ein drittes Kind aufmachen. Im Schnitt, so sagen die Zahlen der Statistik, müssen die Eltern von drei Kindern gut 4000 Euro netto mit nach Hause bringen, um ihren Lebensstandard zu halten. Rund 500 bis 700 Euro mehr als mit zwei Kindern. Viele Paare schaffen das nicht, deshalb ist in Großfamilien das Armutsrisiko höher. Vereinfacht lässt sich sagen, dass jeder fünfte Haushalt mit drei Kindern in Armut lebt (...)“. N. Oberhuber, Können wir uns ein drittes Kind noch leisten?, http://www.faz.net/aktuell/finanzen/meine-finanzen/familien/familienplanung-koennen-wir-unsein-drittes-kind-noch-leisten-12596305.html (10.10.2013).

18 „In Deutschland fällt beispielsweise eine Familie mit einem Durchschnittseinkommen von knapp unter 30.000 Euro, einem Verdienst und zwei Kindern in die relative Armut. Das Armutsrisiko 


\section{Politische Benachteiligung der Familie}

Grundsätzlich können wir, im Lichte der dominierenden links-liberalen, konsumorientierten, individualistischen Ideologie auch im politischen Bereich fragen: Sind Familie und Kinder eine Privatsache, die den Staat nichts angeht? Warum forciert der Staat die Erwerbsarbeit von Frauen? Ist das als eine Erfüllung des Postulates nach mehr Gleichberechtigung oder als ökonomischer Zwang zu verstehen? Wenn der Staat sich so verhält, werden damit die Chancen für eine freie Entscheidung der jungen Frauen und Männer eine Familie zu gründen erschwert oder erleichtert?

Aus dem oben Gesagten lässt sich leicht feststellen, dass die Schwäche der gegenwärtigen Politik darin liegt, gegenüber dem dominierenden ökonomischen Denken und den Gesetzen des „freien“ Marktes die Familie trotz aller noch bestehenden rechtlichen Regelungen zu vernachlässigen. Es werden auch immer mehr „Gleichstellungsrechte“ erdacht, die in ihrem Ziel die Institution der Familie relativieren und unterminieren.

$\mathrm{Zu}$ einer gerechten Ordnung der Gesellschaft gehören das Personsein des Menschen als oberster Bezugspunkt und Ziel sowie die Familie als dem Staat vorgegebene Institution. Die Familie, so heißt es in Rerum novarum, sei älter als jedes andere Gemeinwesen und besitze unabhängig vom Staat ihr ,,innewohnende Rechte"19. Dieser Gedanke hat weitreichende Konsequenzen. Die Familie darf nicht nur als ,Reproduktionsinstanz' gesehen werden.

Familienpolitik, die das Wohl der Familie im Blick hat, versucht die sozialen Strukturen so zu beeinflussen, dass die Schwierigkeiten für Familien gemindert werden und die Erfüllung ihrer Aufgaben eine familiengerechte Unterstützung findet.

Diese durchgängige Überzeugung vom (Vor-)Rang der Familie wird in unserer Gesellschaft weitgehend geteilt. Sie ist die geistige Grundlage für Art. 6 des Grundgesetzes (,Ehe und Familie stehen unter besonderem Schutze der staatlichen Ordnung"). Jugendstudien zeigen immer wieder, dass verlässliche Partnerschaft und Familie mit Kindern ganz oben auf der Werterangordnung junger Menschen stehen.

von Familien mit mehreren Kindern ist erheblich, um gar nicht vom Armutsrisiko Alleinerziehender zu sprechen, das noch höher ist"“. E. Mack, Christliche Familienethik in einer Zeit gesellschaftlichen Wandels, „Theologie der Gegenwart“ 48 (1/2005), S. 19.

19 Rerum Novarum 9. 
- Ein besonderes Problem sind die Wohnverhältnisse. Mit steigender Kinderzahl wächst die durchschnittliche Unterversorgung mit Wohnraum.

- Die Rechte der Familie auf die Erziehung ihrer Kinder werden ignoriert: „Wenn wir erfahren, dass in Londoner Kindergärten und in schwedischen, die als besonders fortschrittlich gelten, der Gebrauch der Worte „Vater" und „Mutter" durch die Betreuer verboten ist und durch geschlechtsneutrale Worte ersetzt wird - aus österreichischen Amtsstuben wird Ähnliches berichtet - dann schwanken in der Regel die Gefühle zwischen Kopfschütteln und Empörung, vor allem weil das Volk seine Vertreter niemals dazu legitimiert hat, von ihnen umerzogen zu werden “20.

- Die Wahlrechte der Familie werden den Singles und Kinderlosen gleichgesetzt und dem individualistischen Bild der Gesellschaft angepasst ${ }^{21}$.

- Das Sozialsystem begeht, laut Franz Xaver Kaufmann, den zentralen Konstruktionsfehler ${ }^{22}$, der sich durch einen „,von Jahrgang zu Jahrgang steigenden Anteil kinderloser Frauen und Männer" räche ${ }^{23}$.

- Die Gesellschaft polarisiere sich immer mehr in Familien mit überwiegend zwei und mehr Kindern auf der einen und kinderlosen Lebensformen auf der anderen Seite. Zwischen beiden herrsche eine ,gravierende Form sozialer Ungleichheit".

- Die bedrohlich niedrige Geburtenrate (z.B. in Deutschland oder in Polen) sei nicht nur Folge von veränderten kulturell-bedingten Lebenseinstellungen, sondern eines Systems, das Kinderlosigkeit geradezu bevorzugt und belohnt.

- Der Sonntag wird immer weniger geschützt ${ }^{24}$.

${ }^{20}$ R. Spaemann, Geleitwort, in: G. Kuby, Die globale sexuelle Revolution, Regensburg 2012, S. 14.

${ }^{21}$ Mehr dazu: M. Spieker, Mehr Kinder oder mehr Erwerbstätige?, http://www.bpb.de/apuz/30652/ mehr-kinder-oder-mehr-erwerbstaetige?p=all (13.09.2013).

22 „Wilfried Schreiber, der Vater der Rentenreform von 1957, war sich bewusst, dass der Generationenvertrag eigentlich kein Zwei-, sondern ein Drei-Generationen-Modell ist. Die aktiv Erwerbstätigen kommen für die Nicht-mehr-Erwerbstätigen und die Noch-nicht-Erwerbstätigen auf, also für Rentner und Pensionäre ebenso wie für Kinder. Dementsprechend schlug er vor, neben der Alterskasse eine ebenfalls umlagefinanzierte Kinderkasse einzurichten. Bei der Alterskasse ist das in Form der gesetzlichen Rentenversicherung geschehen, bei der Kinderkasse nicht"“. H. Kues, Arbeit und Familie, http://www.kas.de/upload/dokumente/2013/Zukunft_der_Arbeit/zukunft_der_arbeit_kues. pdf (13.09.2013).

${ }^{23}$ F-X. Kaufmann, Herausforderungen des Sozial-staats, Frankfurt 1997, S. 79.

${ }^{24}$ Das klassische Kirchenjahr, das den Rhythmus der bäuerlichen vorindustriellen Gesellschaft bestimmte, kannte neben den Sonntagen rund fünfzig Feiertage. Auch die Werktage unterlagen einem festen Rhythmus von Arbeits- und Ruhezeiten. Das christliche Menschenbild fordere 
- Experimente in Bildungsprogrammen ${ }^{25}$, (die Genderideologie wird gegen das subsidiäre Selbstbestimmungsrecht der Eltern und Nationen global propagiert).

\section{Familie nach Karol Wojtyla/Johannes Paul II.}

\section{Der Mensch und seine Würde}

Die entscheidende Frage unserer Kultur ist, nach der Auffassung Johannes Pauls II., die Verteidigung und Wiederentdeckung der menschlichen Person und ihrer Bestimmung zur Transzendenz. Die verengte Auffassung des Menschen, die sich als wissenschaftlich und rational bezeichnet und sich lediglich auf die Argumente von relativistischen Theorien stützt, greift nach Auffassung des Papstes zu kurz. Einerseits wurde der Mensch anknüpfend an die Offenbarung (theologische Argumentation) durch die unauslöschbare Würde (zu objektiver Wahrheit, Schönheit und Güte) bestimmt, andererseits, dank der rein rationalen Denkweise (philosophische Argumentation), zeigt sich der Mensch durch seine Fähigkeit zur Selbstbestimmung und zu moralischen Handlungen, (durch seine Entscheidungen und freien Taten), in seiner wahren Größe, die ihn von anderen Lebewesen unterscheidet.

Die gegenwärtige Herabstufung des Menschen durch den relativistischen Dogmatismus unserer dominierenden postmodernen Kultur bildet die Hauptursache unserer gegenwärtigen Krisen und ist in ihrem Kern ein Ringen um das Wesen des Menschen. Die materialistischen Auffassungen vom Menschen sind die Folge eines falschen Bezuges zur Wahrheit und damit eines „anthropologischen Fehlers“. Ist der Mensch absolut autonom und lediglich vor sich selbst verantwortlich? Oder steht er mit seinen moralischen Entscheidungen nicht nur vor sich selbst, sondern der selbstoffenbarenden Wahrheit, Schönheit und Güte (die er nicht nur durch den Glauben, sondern auch durch die Vernunft erkennen kann) gegenüber? Wenn die Antwort individualistisch ausfällt bleibt, der Mensch einsamer Kreator seiner Wirklichkeit und damit wird auch das

,Unterbrechungen' des Arbeitsalltags, damit der Mensch zu seinen persönlichen, familiären und spirituellen Wurzeln (vgl. Caritas in veritate Nr. 63) zurück finden könne.

${ }^{25}$ Z.B. in einigen fortschrittlichen Kindergärten in Schweden oder England werden die Kinder aufgefordert eine andersgeschlechtliche Rolle einzunehmen, Jungen sollen mit Puppen spielen, Mädchen mit Autos. Ein anderes Beispiel sind die Absichten ein drittes, neutrales Geschlecht im Pass eintragen zu lassen. 
Postulat der Solidarität in seinen tiefen Wurzeln seines Anspruches beraubt. Der Versuch, lediglich auf dem Humanismus basierend, solidarisch zu handeln erweist sich, nach Auffassung des Papstes aus Polen, als mangelhaft. Laizistische, säkulare Menschenbilder leiden in ihrem Kern an einer inneren Schwäche, die dem Missbrauch und der Manipulation oder sogar der Gewalt von Menschen gegenüber anderen Menschen die Türe öffnen. Wenn es keine objektive und verpflichtende Würde der Person gibt und alles, was wir über den Menschen in seinen ethischen Postulaten sagen können, subjektiv bleibt, bereiten wir der Willkür und der Macht des Stärkeren den Weg. In ihrem Wesen leidet unsere Kultur an der Absenz Gottes, der nach Johannes Paul II. die notwendige Quelle und der Anwalt der Würde jedes Menschen ist.

Johannes Paul II. erlebte zwei Totalitarismen. Die Ideologie des Nationalsozialismus und auch die marxistische Fortschrittsideologie des Kommunismus haben die einzelne Person zum Opfer gemacht. Im Grunde gilt das schwächere Subjekt bei den beiden Ideologien wenig. Es kann den ,größeren Zielen“ geopfert werden, was auch tatsächlich geschehen ist. Die Grausamkeiten des Kommunismus, der nicht weniger Menschenleben als der Faschismus kostete, scheinen selbst 60 Jahre nach dem Krieg in unserer links-liberalen Elite nicht ganz durchschaut $\mathrm{zu}$ werden. Es mag als Beispiel das marxistisch fundierte und mit Hilfe der technischen und auch finanziellen Möglichkeiten geschickt propagierte Programm der Genderideologie genügen, um zu erkennen, dass die giftigen Ideen, die bereits im 20. Jh. ihren Tribut forderten, weiterhin in neuen Formen leben. Der Papst aus Polen erkannte richtig, dass die Schlüsselfrage unserer Gegenwart anthropologisch fundiert ist. Er verteidigte das Menschenbild, das einerseits an die Ebenbildlichkeit Gottes (Gen 1,27) anknüpft, andererseits die Fundamente der gegenseitigen Achtung und Befähigung zur Liebe und Solidarität in der Würde der Person begründet.

Diese innere Würde des Menschen verstehen wir, laut Wojtyla, wenn wir den moralischen Imperativ zur verantwortlichen Liebe und Achtung praktisch anerkennen und in die Tat umsetzen. Deshalb hebt er im Titel seines Hauptwerkes die Person und die Tat hervor. Die Solidarität in dieser ersten Dimension (individuell) bedeutet also zuerst die eigene Würde, sowie die Würde des anderen (jedes Menschen als Person!) anzuerkennen und danach zu handeln. Die gegenwärtige Krise wurzelt in dieser mangelhaften Anerkennung und Achtung gegenüber jeder Person. Obwohl viel über Individualismus und die individuellen Rechte gesprochen wird, bleiben sie unverbindlich in der Luft hängend, subjektiv begründet. Wojtyla sah mit Recht diese innere Schwäche 
der dominierenden Ideologien, die durch ihre materialistische Auffassung des Menschen nicht nur die Rechte vor allem der Schwächeren missbrauchen können, sondern in Bezug auf unser Problem der Familie, die moralische Verpflichtung in Frage stellen. Eine wirklich humane Gesellschaft lässt sich nur auf der Basis eines verbindlichen Ethos errichten. Die sicherste Verankerung eines Ethos liegt in der „transzendenten“ Begründung der Menschenwürde: „Wenn es keine transzendente Wahrheit gibt, der gehorchend der Mensch zu seiner vollen Identität gelangt, gibt es kein sicheres Prinzip, das gerechte Beziehungen zwischen den Menschen gewährleistet ${ }^{\text {“26. }}$.

\section{Die Familie als Berufung zur Liebe und Verantwortung}

In seinem Werk „Liebe und Verantwortung“, publiziert 1960, bezieht sich der Autor auf die Reihe seiner Vorlesungen an der katholischen Universität in Lublin und auf seine pastoralen Erfahrungen mit jungen Familien. Wie Yves Semen in seinem Buch „Die Familie nach Johannes Paul II.“ erinnert, zelebrierte der künftige Papst schon als Kaplan sehr viele Hochzeiten ${ }^{27}$, er entwickelte eine pastorale Vorbereitung auf das Sakrament der Ehe und die Thematik von Ehe und Familie stand im Vordergrund seines pastoralen Handelns. Auch als Papst Johannes Paul II. beruft er bereits im Jahre 1981 ein Pontifikalkonzil für die Familie. Im gleichen Jahr erscheint auch „Familiaris consortio“. Er gründete ein Institut für die Belange der Familie und als erster Papst sprach er ein Ehepaar, die Eheleute Luigi und Maria Beltrame-Quattrocchi, selig ${ }^{28}$. Es ist der erste Fall in der Geschichte der Kirche, wie Semen feststellt, dass ein Papst Eheleute, aufgrund der Heiligkeit ihrer Ehe, zur Ehre der Altäre erhob ${ }^{29}$. Warum so spät? Warum wurde z.B. die Familie von Jozef und Wiktoria Ulma

${ }^{26}$ Centesimus annus 44, 2.

27 Seite 13: schon als Vikar in Niegowic spendete er in 8 Monaten 30 Hochzeiten. Als Vikar in St. Florian in Krakau mehr als eine Hochzeit pro Woche. Y. Semen, La familia secondo Giovanni Paolo II, Milano 2012, S. 13.

28 ,In ihrem Leben wie in dem vieler anderer Ehepaare, die sich bemühen, Tag für Tag ihren Pflichten als Eltern nachzukommen, kann man beobachten, wie die Liebe Christi zur Kirche sakramental aufscheint. Denn die Eheleute erfüllen »in der Kraft dieses Sakramentes ihre Aufgabe in Ehe und Familie. Im Geist Christi, durch den ihr ganzes Leben mit Glaube, Hoffnung und Liebe durchdrungen wird, gelangen sie mehr und mehr zu ihrer eigenen Vervollkommnung, zur gegenseitigen Heiligung und so gemeinsam zur Verherrlichung Gottes« (Gaudium et spes, 48)“. http://www.vatican.va/holy_father/ john_paul_ii/homilies/2001/documents/hf_jp-ii_hom_20011021_beltrame-quattrocchi_ge.html (17.09.2013).

${ }^{29}$ Vgl. Y. Semen, La familia secondo Giovanni Paolo II, Milano 2012, S. 52. 
aus Polen ${ }^{30}$ immer noch nicht zur Würde der Altäre erhoben? Das zeigt indirekt eine gewisse Vernachlässigung der Theologie des Leibes ${ }^{31}$ der bisherigen kirchlichen Lehre, die der polnische Papst zu korrigieren versuchte. Diese immer noch andauernde Leibfeindlichkeit der offiziellen Lehre der Kirche ${ }^{32}$, welche die Spuren der manichäischen Abwertung trug, suchte der Papst aus Polen aufgrund seiner Erfahrungen und Lehre zu verändern. Schon die Überbetonung des Zieles der Ehe in der klassischen Lehre auf die Erzeugung der Nachkommen, verrät eine quasi funktionelle Auffassung der Leiblichkeit, die lediglich zum Zwecke der „Produktion“von Nachkommen dienen sollte, der Liebesbeziehung der Eheleute an sich aber keinen eigenen Wert beimaß. Bei Johannes Paul II. wird die eheliche Liebe im Bild der communio personarum Gottes, als die vollkommenste Form der Liebe präsentiert. Damit bemüht er sich, diese verkürzte spiritualistische Sicht menschen- und familiengerecht darzustellen. In der Philosophie des Papstes spielt die Ich-Du-Erfahrung der Liebe eine außerordentliche Rolle ${ }^{33}$. Wojtyla beschreibt verschiedene Stadien der Liebe ${ }^{34}$, die sich auf die vollkommene Hingabe hin entwickeln soll und sich in reifer Form wohlwollend als Gabe an die geliebte Person verschenkt. An Semen anknüpfend können wir drei wichtige Schwerpunkte, die neue Akzente in der Lehre über die eheliche Liebe setzen, erkennen: Der erste ist, wie bereits oben erwähnt, die eheliche Liebe als vollkommenste Form der Liebe. Die zweite

${ }^{30}$ Es ist wichtig zu bemerken, welche Versuche gemacht wurden diese Familie zu würdigen: Auf der Ebene der Erzdiözese begann der Beatifikationsprozess im Jahre 2003, abgeschlossen war er im Jahre 2008. Drei Jahre später wurde die Dokumentation an den Vatikan geschickt. Siehe: M. Kamieniecki, Muzeum pokaże prawdę, http://www.naszdziennik.pl/polska-kraj/56375, muzeum-pokaze-prawde. html. „Rozpoczęty w 2003 r. na szczeblu archidiecezji proces beatyfikacyjny Ulmów zakończył się w 2008 roku. W 2011 r. dokumentacja trafiła do Watykanu”. „Muzeum będzie nosiło imię Sług Bożych rodziny Józefa i Wiktorii Ulmów, którzy wraz z sześciorgiem dzieci zostali zamordowani przez Niemców w 1944 r. za ukrywanie ośmiorga Żydów z rodzin Szallów i Goldmanów“. Ebenda. http://www.naszdziennik.pl/polska-kraj/56375,muzeum-pokaze-prawde.html (11.10.2013).

${ }^{31}$ Siehe mehr dazu: M. Groos, T. Loichen, M. Gerwing (Hg), Liebe, Leib und Leidenschaft. Zur Theologie des Leibes von Johannes Paul II., Medienverlag 2012.

${ }^{32}$ Noch im Jahre 2009, anlässlich der ersten Seligsprechung der Familie durch den Papst Benedikt XVI. wurde ein weiteres Faktum aus dem Leben der neuen Seligen durch den Prälat Prof. Dr. Helmut Moll hervorgehoben: „Louis Martin hegte beim Schließen der Ehe die Hoffnung, mit seiner Gattin eine rein geschwisterliche Bindung einzugehen und wie Bruder und Schwester zusammenzuleben“. H. Moll, Zur Seligsprechung der Französischen Eheleute Louis und Marie Zelie Martin, http://www. familientrends.de/index.php?option $=$ com_content\&task=view\&id=429\&Itemid=1 (17.09.2013).

${ }_{33}$ Z. Waleszczuk, Gleichheit der Rechte und Pfichten im Denken Karol Wojtylas, „Logos und Ethos“" 1 (2013) Nr. 34, S. 81.

${ }^{34}$ Z. Waleszczuk, Globalizacja solidarności, Wrocław 2007, S. 256-261. 
knüpft an den kategorischen Imperativ von Immanuel Kant an und mündet in der Formulierung der personalistischen Norm. Lieben steht in Opposition zum Nutzen. Der dritte Schwerpunkt in Wojtylas „Liebe und Verantwortung“ ist die Affirmation der Liebe als Norm der Ehe. Die klassische Auffassung betonte zu stark die Nachkommen zeugende Aufgabe der Eheleute. In „Liebe und Verantwortung"wendet sich Wojtyla bewusst zurück an die beiden sich liebenden Personen. Im Zentrum seiner Ausführungen steht die Gabe und Aufgabe zur Liebe, die auf der Würde der Person basiert. In seiner personalistischen Norm geht es um eine Verpflichtung gegenüber dem anderen Du. Wenn sich der Mensch lediglich von den Naturnotwendigkeiten in allen seinen Handlungen und Entscheidungen und durch seine sinnlichen Wünschen leiten lässt und dazu neigt den anderen lediglich zu benutzen, bleibt er in seinem Egozentrismus verhaftet. Hier herrscht keine Freiheit und damit auch keine Liebe. Erst dort, wo der Mensch erkennt, was das moralische Gesetz gegenüber dem anderen $\mathrm{Du}$, sowie auch gegenüber sich selbst als Person verlangt, als rechtmäßigen Anspruch auf Achtung, erst dann beginnt die freie und verantwortliche Liebe - in der Tat, die wohlwollend das Gute der anderen Person, sowie auch seiner selbst anstrebt. Die wahre Freiheit ist erst dann möglich, wenn der Mensch zwischen Gut und Böse unterscheiden kann. Die verantwortliche Liebe bedeutet die Fähigkeit sich selbst zu verschenken, sich als eine Gabe für den anderen zu verstehen. „Die Person“ - schreibt Wojtyla in „Liebe und Verantwortung“ - , ist ein objektives Wesen, das als bestimmtes Subjekt mit der ganzen (äußeren) Welt eng in Verbindung steht und dank seiner Innerlichkeit und seines Innenlebens darin von Grund auf wurzelt. Dazu kommt, dass die Person so nicht nur mit der sichtbaren Welt in Verbindung steht, sondern auch mit der unsichtbaren Welt und vor allem mit Gott. Dies ist ein weiteres Anzeichen dafür, dass die Person in der sichtbaren Welt etwas ganz Besonderes ist ${ }^{\text {“35. }}$.

„Nur Personen haben an der Liebe teil'“36, betont Wojtyla, damit unterstreicht er auch die Beziehung des Menschen zu seinem Schöpfer, die ähnlich erfahren wird wie zu einem anderen Menschen. Das Verlangen nach wahrer Liebe realisiert sich in der Befähigung zur personalen Kommunikation: ich - du.

Die Rolle des Glaubens im Prozess der Teilhabe an der Liebe fand Wojtyla beim heiligen Johannes vom Kreuz, der mit einer Metapher die Beziehung zwischen Glauben und Vernunft beschrieb. Die Vernunft wird mit einem Kerzenständer

${ }^{35}$ K. Wojtyła, Liebe und Verantwortung. Eine ethische Studie, München 1981, S. 22.

${ }^{36}$ K. Wojtyła, Osoba i czyn, Lublin 2000, S. 131. 
verglichen. Erst dank der Kerze des Glaubens kann das Licht der liebevollen Vereinigung mit Gott als Licht der Liebe aufleuchten. Wieso ist die Dimension der liebevollen Kommunikation mit Gott so relevant für die eheliche Beziehung nach Karol Wojtyla? Implizit befindet sich in der personalistischen Norm das moralische Gesetz, das einen Ruf an unser Gewissen enthält. Jeder Mensch der sich ausschließlich auf die völlig autonome, subjektive, utilitaristische Auffassung der Wirklichkeit einlässt, behindert damit seine Bestimmung zur Liebe. Als ens sociale sind wir auf die Hilfe der Familie angewiesen. In seiner Lehre betonte Johannes Paul II. den dringenden Bedarf einer Aufwertung der Familie. Seiner Meinung nach ist sie eine Gabe und ein Gut für alle. Der Papst erinnert und vertieft die Theologie der Ehe, er lehrt uns, was die eheliche Liebe als Tat der gegenseitigen Hingabe und communio bedeutet. Die Gesellschaft braucht eine gesunde, im Glauben starke Familie als communio personarum. Die gesunde Familie ist eine Widerspiegelung der Gottesliebe. Die Familie ist bei Johannes Paul eine Schule des echten Humanismus, die jeden Menschen befähigt auch in größeren Gemeinschaften die Mühe der Solidarität zu verwirklichen.

\section{Familie als Schule der Solidarität}

Nach Johannes Paul II. ist die Familie das erste Subjekt und Objekt der Solidarität. Eine besondere Stellung in dieser Auffassung nimmt die Solidarität der Arbeit ein. Die menschliche Arbeit ist eine Realisierung der Berufung des Menschen. In seinem Wesen ist der Mensch zur Kooperation bestimmt. Er muss auf der vertikalen Ebene gemeinsam handeln, aber auch in der Beziehung zu Gott verbleiben, um mehr Mensch zu werden. Die Arbeit ist nicht nur bonum arduum - mit der Mühe verbunden, sondern auch bonum honestum, etwas, das den Menschen erhebt und ihm zum Segen wird ${ }^{37}$. Die besondere Rolle der Familie auch in Bezug auf die Vorbereitung zur Kooperation, zur Übernahme von Verantwortung für andere Personen, Institutionen usw. wird heutzutage leider stark unterschätzt.

Das Ausblenden der transzendenten Wahrheit über den Menschen wirkt sich in Richtung einer egoistischen Selbstentfremdung aus, was auch eine gerechte Ordnung des Sozialen und Politischen gefährdet. Dies zeigt sich in einer Tendenz zu einem Klassen- oder Gruppenegoismus, der die Menschen jeweils „in Gegensatz zueinander“ bringt. Wie Johannes Paul II. schreibt: Wenn wir die

\footnotetext{
${ }^{37}$ Laborem exerzens 9.
} 
Perspektive der gleichen Würde aller Menschen (und damit der MenschheitsFamilie) verlassen, ,wenn die transzendente Wahrheit nicht anerkannt wird, dann triumphiert die Gewalt ${ }^{\star 638}$. Nur wenn die Würde des Menschen in einer „transzendenten Wahrheit“ gründet, ist der Mensch „Subjekt von Rechten, die niemand verletzen darf: weder der Einzelne noch die Gruppe, die Klasse, die Nation oder der Staat ${ }^{\text {“39. }}$.

Der Papst aus Polen schreibt über die Rolle der Familie folgendermaßen: Die „auf die Ehe gegründete Familie“ ist es, in der die ,gegenseitige Hingabe von Mann und Frau eine Lebensatmosphäre schafft, in der Kinder geboren werden und ihre Fähigkeiten entfalten können. In ihr werden sie sich ihrer Würde bewusst und können sich auf ihre einmalige und unwiederholbare Bestimmung vorbereiten ${ }^{\star 40}$. Leider, kann man die Familie bedrohen, so Johannes Paul II., sofern man „der Behauptung“ zuneigt, „der Agnostizismus und der skeptische Relativismus" seien die Philosophie und die Grundhaltung, die den demokratischen Formen des politischen Lebens entsprechen. Alle, die überzeugt sind, die Wahrheit zu kennen, und an ihr festhalten, seien vom diesen Standpunkt her nicht vertrauenswürdig, weil sie nicht akzeptieren, dass die Wahrheit von der Mehrheit bestimmt werde ${ }^{641}$. Das demokratische Mehrheitsprinzip bietet dann kein Hindernis gegen eine möglicherweise die Menschenrechte verletzende „Diktatur“ der Mehrheit, wenn „keine letzte Wahrheit anerkannt wird, die das politische Handeln leitet und ihm Orientierung gibt“. Insofern verwandelt sich ,eine Demokratie ohne Werte ... leicht in einen offenen oder hinterhältigen Totalitarismus ${ }^{642}$.

Der dritte Kultursachbereich, in dem die Selbstentfremdung des Menschen droht, ist in der heutigen geistigen Situation zu sehen, denn es geschehe oft, dass der Mensch entmutigt wird, ,die naturgegebenen Bedingungen zur Weitergabe des Lebens zu schaffen". Er lässt sich dazu verleiten, sich selbst und sein Leben ,als eine Folge von Sensationen zu betrachten, die es zu erleben, nicht aber als eine Aufgabe, die es zu erfüllen gilt ${ }^{643}$. Hier spricht der Papst

\footnotetext{
38 Centesimus annus $44,2$.

39 Centesimus annus 44, 2.

${ }^{40}$ Centesimus annus 39, 1.

${ }^{41}$ Centesimus annus 46, 2.

${ }^{42}$ Centesimus annus 46, 2.

${ }^{43}$ Centesimus annus 39, 1.
} 


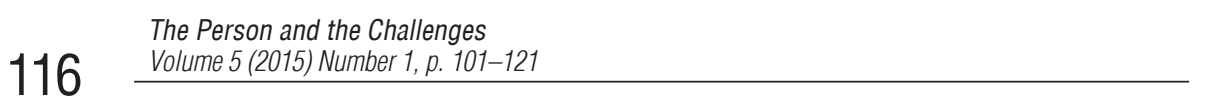

genau jene Phänomene an, die in der heutigen Kultursoziologie zum Begriff „Erlebnisgesellschaft" (Gerhard Schulze) geführt haben.

Scheinbar führt die Erlebnisgesellschaft zu immer mehr „Identität“ des Menschen mit sich selbst und seiner Welt. In Wirklichkeit aber entsteht daraus „ein Mangel an Freiheit, der die Verpflichtung, sich endgültig an einen anderen Menschen zu binden und Kinder zu zeugen, ablehnt oder dazu verleitet, Partner und Kinder als eines der vielen ,Dinge' anzusehen, die man, je nach eigenem Geschmack, haben oder nicht haben kann und die mit anderen Möglichkeiten in Konkurrenz treten ${ }^{\star 44}$. In der heutigen Bedrohung der Integrität der Familie sieht Johannes Paul II. die verhängnisvollste Dimension der Selbstentfremdung des modernen Menschen, die er auf die Kurzformel „Kultur des Todes“ bringt. Dem gegenüber stelle die Familie „den Ort der Kultur des Lebens dar' ${ }^{645}$. Weil dies so ist, darum gehören zu den Menschenrechten nicht nur die individuellen, sondern auch die korporativen Rechte. Der Papst zählt deshalb zu den ,vorrangigsten Rechten ... das Recht auf Leben, zu dem wesentlich das Recht gehört, nach der Zeugung im Mutterschoß heranzuwachsen“ sowie „,in einer geeinten Familie und in einem sittlichen Milieu zu leben, das für die Entwicklung und Entfaltung der Persönlichkeit des Kindes geeignet ist ${ }^{646}$.

\section{Familie das Fundament der Zukunft - wie kann und soll sie unterstützt werden?}

Diese Frage ist nicht nur für die Kirche, sondern aufgrund der Zukunft jedes Menschen und der ganzen Menschheitsfamilie sehr relevant. Es ist nicht nur für die Sozialethik der Kirche, sondern auch für den Staat eine Existenzfrage. Der polnische Philosoph Kolakowski referierte schon 1993 in Berlin über den Relativismus ${ }^{47}$. Er sprach über die Konsequenzen des Kommunismus,

${ }^{44}$ Centesimus annus 39, 1.

${ }^{45}$ Centesimus annus 39, 1.

${ }^{46}$ Centesimus annus 47, 1.

${ }^{47}$ Kolakowski sprach von einem „populären Relativismus“, der die „Säulen, auf welche sich unsere Zivilisation stützt ... konsequenter als der Kommunismus“ zerstöre, weil er uns von jeder Verantwortung und Pflicht befreie. Eine Gesellschaft, die Freiheit und sonst nichts postuliere, stehe in der Gefahr, letztlich in ihr Gegenteil umzuschlagen; denn „,am Ende jeder Anarchie wartet ungeduldig die Tyrannei“. Kolakowski warnt vor dem Aberglauben, dass Freiheit von selbst zu einer Ordnung führe. ,Für Kolakowski ist der entscheidende Punkt, daß es letztlich auf den „Glauben an 
die unsere Zivilisation zerstören, er sprach über die Verantwortung und das Pflichtbewusstsein und die Tendenz unserer Kultur, den Menschen von seinem Pflichtbewusstsein zu „,befreien“. Besonders interessant ist seine Betonung der Bedeutung des Glaubens an die sinnvolle Ordnung dieser Welt, die außerhalb der religiösen Tradition nicht zu finden ist. In diesem Punkt stimmt die Diagnose des Agnostikers mit dem Denken Johannes Pauls II. überein. Wie wir oben dargestellt haben, konzentriert sich die Lehre des Papstes aus Polen auf die kulturellen Fragen des falschen Menschenbildes, die er als Ursache der Krisen, auch der Krise der Familie betrachtet. Seinen Ausführungen und seiner Lehre über die Theologie des Leibes sollte man deshalb mehr Aufmerksamkeit widmen und diese ernsthaft in die Programme der Theologie aufnehmen.

Um wirksame Antworten auf die gegenwärtigen globalen Herausforderungen $\mathrm{zu}$ formulieren, benötigen wir neben dem kulturellen Bereich, auf den sich die Lehre der Kirche meistens konzentriert, praktische Lösungsvorschläge in allen Dimensionen: sozial, politisch und ökonomisch. Man darf diese Felder nicht den Gegnern des christlichen Glaubens allein überlassen. Hier ist der Platz für die engagierten Laiengläubigen. Um die Familie wirksam zu unterstützen, reicht heute der Nationalstaat allein nicht aus, sondern durch die Globalisierungsprozesse brauchen wir neue multinationale Regelungen. Obwohl die politische und ökonomische Dimension in der Diagnose der gegenwärtigen Krisen separat behandelt wurden, lassen sie sich im praktischen Bereich nicht trennen, denn die Ökonomie ist abhängig von der Politik und umgekehrt. Bevor wir auf die globalen Vorschläge eingehen, möchte ich einige konkrete Beispiel erwähnen, die bereits in lokalen Bereichen umgesetzt wurden:

- Viktor Orban, der Premierminister von Ungarn, zwang die Banken mehr Steuern zu zahlen um dadurch vor allem kinderreichen Familien eine Steuererleichterung gewähren zu können. Heute zahlt eine Familie mit drei Kindern in Ungarn praktisch keine Steuern mehr. Es ist ein konkretes Beispiel der ökonomischen Entlastung der Familie, die aus Sicht der christlichen Sozialethik zu begrüßen ist. Leider - oder wie zu erwarten - wird diese politische Neuregelung durch die einflussreichen Medien Europas nicht begrüßt, vielmehr scheut man sich nicht davor, ökonomischen, politischen und medialen Druck einzusetzen, um diese Regierung zu stürzen. 
- Durch die Währungsspekulationen, meistens mittels schweizerischer Franken, wurden sehr viele Familien in Spanien, Kroatien, Ungarn, Polen etc. verschuldet. Es gibt Gerichtsurteile (z.B. in Spanien), die dieses Vorgehen der Banken als schuldhafte Spekulation entlarven. Leider, wie im oben gezeigten Beispiel, scheinen die Machtverhältnisse deutlich ungleichmäßig zu sein. Noch bevor die Urteile rechtskräftig wurden, erhoben sich laute Proteste in der Medienwelt. Selbstverständlich werden die bestbezahlten Anwälte und auch korrupte Politiker alles tun, um die Interessen der Banken durchzusetzen. Es geht um Milliarden Profite, die, falls solche Urteile Schule machen, für das Gemeinwohl verwendet werden könnten, anstatt private Taschen zu füllen. Es geht um den Status quo, bei dem nicht der Staat und die Politik, sondern die Banken und das Kapital das Sagen haben.

- Die Ökonomisierung des öffentlichen Lebens trägt dazu bei, dass alle Bereiche nach ökonomischen Maßstäben beurteilt werden. Die einzige Möglichkeit, eine Stabilisierung zu erreichen und dem Drama der Familienkrise entgegenzuwirken, besteht darin politische Regelungen in globalen Dimensionen durchzusetzen, welche die Willkür des Finanzkapitalismus bremsen. Ein Beispiel aus der Schweiz: Per Volksbegehren wollten die Bürger durchsetzen, dass ein Manager maximal ein Jahresgehalt des durchschnittlichen Arbeitnehmers der gleichen Firma monatlich verdienen darf. Unter größter Mühe der Einflussnahme und einer nicht verborgenen Erleichterung haben die Medien aufgeatmet, nachdem die notwendige $50 \%$ Mehrheit knapp verfehlt wurde ${ }^{48}$.

- Ein wichtiger Punkt für die Förderung der Familie ist die humane Bildung, die leider oft vernachlässigt wird. Es werden technische Fächer allgemein favorisiert und das Humanwissen benachteiligt.

- Je mehr die Religion als reine Privatsache an den Rand der Gesellschaft abgedrängt wird und im Zentrum offiziell neutrale, in der Praxis aber materialistische und atheistische Menschenauffassungen stehen, desto schwieriger wird es wirksame Gesetze zur Förderung der Familie zu schaffen.

48 „Fast drei Viertel der Deutschen sprechen sich dafür aus, Topmanagern die Gehälter zu beschneiden. Das zeigt eine repräsentative Umfrage des Marktwirtschaftsinstituts GfK. Anlass war die sogenannte $1: 12$ in der Schweiz. Auf die Frage, ob sie für eine solche Regelung seien, antworteten 73,3 Prozent der Befragten zustimmend“. Schweizer lehnen Gehaltbremse ab, Frankfurter Neue Presse, S. 4. 
- Ein anderes Postulat hat Manfred Spieker formuliert - er spricht von einem Familienwahlrecht - d.h. jedes Kind hat auch politisches Wahlrecht, das die Eltern im Namen des Kindes ausüben dürfen, damit wäre das Gewicht der Familie aufgewertet ${ }^{49}$.

- Der deutsche Sozialethiker spricht auch über den Bedarf flexiblerer, angepasster Arbeitszeiten für berufstätige Frauen. Es geht um Erleichterungen und Entlastungen bei der Erziehung der Kinder. Leider läuft momentan alles nur in die eine Richtung, dass mehr Kinderkrippen gebaut und mehr Erzieherinnen beschäftigt werden, so als ob die einzige Möglichkeit der Familie zu helfen darin bestehen würde, das Kind möglichst schnell bei einer staatlichen Erziehungsanstalt abzugeben. Dieses Modell, trotz aller liberal klingenden Slogans, verbirgt eine totalitäre Absicht nach dem DDR-Modell. Trotz äußerlicher Parolen, dass man der Familie helfen will, geht es im Kern um möglichst frühzeitige Erziehung der Kinder im Sinne der Genderideologie. Solche Programme werden global stark finanziell und politisch gefördert.

- Die dramatische Situation der Familien ist auch oft mit fehlendem Wohnraum verbunden. Wenn sich junge Leute keine eigene Wohnung leisten können, ist es kein Wunder, dass sie vor allem nach einem Ort suchen, wo sich der Traum einer eigenen Familie zu erfüllen vermag ${ }^{50}$. Die polnischen Frauen, die in England oder Irland leben, entscheiden sich zweimal häufiger für Kinder als diejenigen, die in Polen verbleiben. Aus diesem Beispiel ist ersichtlich, dass es in vielen Ländern mangelhafte ökonomische und politische Anreize gibt, um eine Familiengründung zu wagen. Im Gegenteil ist eine Entscheidung für Kinder immer noch mit einem Armutsrisiko verbunden.

49 „Wer für die Familie das Bürgerrecht fordert, der muss sich der Frage des Familienwahlrechts stellen. Das Recht, in regelmäßigen Abständen die Regierenden bestimmen und dafür unter mehreren Kandidaten auswählen zu können, ist in der Demokratie das Privileg der Bürgerinnen und Bürger. Dieses Recht muss auch der Familie zuteil werden“. M. Spieker, Mehr Kinder oder mehr Erwerbstätige?, http://www.bpb.de/apuz/30652/mehr-kinder-oder-mehr-erwerbstaetige?p=all (13.09.2013). Vgl. Ursula Nothelle-Wildfeuer, Das Kind als Staatsbürger. Wahlrecht gegen die strukturelle Benachteiligung von Familien?, „Herder-Korrespondenz“ 58 (2004), S. 198ff.

${ }^{50}$ Z.B. verließen in den letzten Jahren 2 Millionen Polen, vor allem junge Menschen, ihre Heimat und emigrierten in den Westen. „Die Polen wandern in den Westen aus. Die Auswanderungswelle scheint größer zu sein, als je zuvor eine polnische Emigration“. R. Dutkiewicz, Eine neue ,große Emigration"?, S. Luft, Beiträge zu Migration und Politik, Nr. 1, Oktober 2007. http://www.stefanluft. de/beitraege_mpl.pdf(13.11.2013). 


\section{Fazit}

Zusammenfassend lässt sich sagen, dass die Zukunft der Gesellschaft von der Zukunft der Familie abhängig ist. Es liegt im Interesse des Staates (der Staatengemeinschaft) alles Notwendige zu tun, um die eigene Existenz zu sichern. Die gegenwärtige Krise der Familie steht, wie oben dargestellt, vor allem mit dem falschen Bild des Menschen und seiner Rolle in der Gesellschaft in Verbindung. In Konsequenz wird egoistisches Trittbrettfahren belohnt, die Entscheidung für die Familie jedoch bestraft. Eine solche Situation erfordert es, diese kulturellen, politischen und vor allem ökonomischen Ungerechtigkeiten zu überwinden. Die Aufgabe der Kirche im sozialen Bereich besteht vor allem darin, die wahren Fundamente der Gesellschaft, d.h. die kulturellen Faktoren (das Bild des Menschen und seiner Würde) in der Öffentlichkeit glaubwürdig bewusst zu machen. Leider beobachten wir heute, wie oben aufgezeigt, nicht nur die Angriffe von außen, sondern auch eigenverantwortete Angriffe von innen, die mit den Vernachlässigungen der sozialen Verantwortung zusammenhängen. Die mangelnde Umsetzung der Christlichen Soziallehre folgt auch aus der Tatsache, dass dieser Disziplin geringe Aufmerksamkeit gewidmet wurde. In der heutigen sich schnell globalisierenden Welt, wo Interdependenz groß geschrieben wird, ist ,jeder verantwortlich für jeden“. Wir brauchen christlich motivierte, gute und kompetente Handlungen im politischen, kulturellen und ökonomischen Bereich, d.h. wir brauchen engagierte Christen, die bereit sind das Licht des Evangeliums in alle Bereiche des Menschenlebens zu bringen. Es war nicht die primäre Absicht dieses Beitrags präzise praktische Lösungen zu formulieren. Gleichzeitig wollten wir uns jedoch nicht lediglich auf moralische Appelle begrenzen und mit rein theoretischen Spekulationen zufrieden geben. Schon die oben aufgezeigten Problemfelder können bei der Suche nach praktischen Antworten auf die gegenwärtigen Herausforderungen der Familie als richtungweisend dienen. Sie bieten, hoffentlich, neue Inspirationen und Orientierungshilfen in der Beurteilung der Lage der Familie, sowie der Wahl entsprechender und guter Maßnahmen. Auch wenn der Artikel in gewissem Sinne unvollendet bleibt, eröffnet er hoffentlich viele Lösungsperspektiven auf der Suche nach mehr „Liebe in der Wahrheit" bei der Gestaltung der Welt von heute zum Wohle der ganzen Menschheitsfamilie. 


\section{Bibliography}

Groos M., Loichen T., Gerwing M. (Hg), Liebe, Leib und Leidenschaft. Zur Theologie des Leibes von Johannes Paul II., Medienverlag 2012.

Kaufmann F.-X., Die Krise der Kirche, Herder 2011.

Kuby G., Die globale sexuelle Revolution, Regensburg 2012.

Kues H., Arbeit und Familie, http://www.kas.de/upload/dokumente/2013/Zukunft_der_Arbeit/ zukunft_der_arbeit_kues.pdf. (13.09.2013).

Luft S., Beiträge zu Migration und Politik, Nr. 1, Oktober 2007 http://www.stefanluft.de/ beitraege_mp1.pdf(13.11.2013).

Mack E., Christliche Familienethik in einer Zeit gesellschaftlichen Wandels, „Theologie der Gegenwart" 48 (1/2005).

Oberhuber N., Können wir uns ein drittes Kind noch leisten?, http://www.faz.net/aktuell/ finanzen/meine-finanzen/familien/familienplanung-koennen-wir-uns-ein-drittes-kindnoch-leisten-12596305.html (01.10.2013).

Peeters M.A., Polityka globalistów przeciwko rodzinie, Warszawa 2013.

Roos L., Zwischen Hoffnung und Angst, „Die Neue Ordnung“ (2005) Nr. 6.

Spaemann R., Geleitwort, in: G. Kuby, Die globale sexuelle Revolution, Regensburg 2012.

Sutor B., Politische Ethik, Paderborn 1991.

Waleszczuk Z., Globalizacja solidarności, Wrocław 2007.

Wojtyła K., Liebe und Verantwortung. Eine ethische Studie, München 1981.

Wojtyła K., Osoba i czyn, Lublin 2000. 
